\section{Animal models of emphysema: the next generations}

\author{
Edward J. Campbell
}

Commentary

Department of Medicine, University of Utah, 410 Chipeta Way, Room 410, Salt Lake City, Utah 84108, USA.

Phone: (801) 585-6192; Fax: (801) 585-3118; E-mail: edward.campbell@hsc.utah.edu.

Niewoehner (1) wrote in 1988 that pulmonary emphysema is one of the most important causes of morbidity and mortality in our modern society, and his statement rings just as true today as it did then. Progress in the intervening years has built on the creation and analysis of animal models for this disease, many of which, including the one described by Zheng et al. (2) in a recent issue of the JCI, exploit technology that could not have been imagined at that time.

Animal models are especially important for slowly progressing diseases such as pulmonary emphysema (3). They facilitate the understanding of complex pathogenetic relationships and allow hypothesis testing. They also have the potential to allow tests of preventive therapies that would be inordinately time consuming or ethically forbidden in humans. A review of past and future generations of animal models will place the contribution of Zheng and colleagues in perspective.

\section{First-generation animal models of emphysema: elastases down the windpipe}

In 1965, Gross et al. described the first reproducible animal model of emphysema by instilling the proteinase papain intratracheally into rats (4). Subsequent studies in a variety of animal species demonstrated that only enzymes with the capacity to solubilize the ECM protein elastin can produce emphysematous lesions. These observations, coupled with the discovery of the enhanced risk for development of emphysema in individuals with $\alpha_{1}$-antitrypsin deficiency (5), led to the proteinase-antiproteinase hypothesis and to modern thinking about the pathogenesis of emphysema. These animal models also showed that repair of injured elastic tissue is important in reducing structural lung injury, and that proteolytic injury can result in secretory cell metaplasia, analogous to that seen in chronic bronchitis. Work with these early models also showed that concomitant cigarette smoke exposure leads to more severe emphysema, and that emphysematous lesions progress for at least 6 weeks following the initial enzymatic insult.

In obvious ways, however, these models did not recapitulate the pathogenesis of the human disease, which is produced over decades by gradual injury to the lung structure, rather than by a single massive injury. Moreover, in the human condition, the putative injurious enzymes are delivered to the lung tissue by viable inflammatory or resident lung cells, rather than as a bolus in solution, and they are often delivered in packets, when granules containing overwhelming concentrations of enzyme translocate to the cell surface (6). For these reasons, the first-generation models were not particularly helpful for the development of preventive strategies.

\section{Second-generation models: genetically-engineered manipulations of enzymes and inhibitors}

Certain naturallyoccurring mutant strains of mice develop emphysema (7), but these animals have a complex and lifelong phenotype which may be complicated by abnormal lung development. However, mice can be developed with either overexpression or targeted deletion of specific proteins. One such animal, a mouse which overexpressed interstitial collagenase (MMP-1), spontaneously developed pulmonary emphysema (8). This model was thought-provoking because it violated the time-honored elastase-injury hypothesis. In the end, however, the most important lesson it taught us was that since the overexpression of collagenase is lifelong, it is not possible to separate developmental abnormalities in the lung from the structural injury in adult lungs that defines emphysema.

Another second-generation model took the opposite approach, employing a targeted deletion of macrophage elastase (MMP-12). These mice developed and grew normally. However, when they were later stressed by an exposure to cigarette smoke that caused the development of emphysema in wild-type mice, they did not develop emphysema (9). This work strongly suggested a role for a metalloproteinase in the pathogenesis of emphysema in human cigarette smokers, and provided a mechanism for future hypothesis-testing and therapeutic intervention.

Zheng et al. (2) took yet another approach to modeling the progression of emphysema. Realizing that human cigarette smokers who develop chronic obstructive pulmonary disease (COPD) have abundant inflammatory cells in the lung parenchyma, they targeted the overexpression of a proinflammatory Th2 cytokine (IL-13) to the airway epithelium using the reverse tetracycline transactivator system and employing a promoter that is active solely in Clara cells. In these mice, IL-13 is overexpressed only when the animals are exposed to tetracycline, thus allowing the investigators to activate overexpression after lung development is complete. The transgenic animals, but not wild-type animals, developed airway and lung parenchymal inflammation and emphysema when exposed to tetracycline after completion of lung development. The inflammatory cell infiltration consisted predominantly of macrophages, lymphocytes, and eosinophils, with few neutrophils. Zheng et al. also noted that the transgenic animals developed mucus metaplasia following induction, reminiscent of that seen in chronic bronchitis. In addition, metalloproteinase inhibition substantially prevented development of emphysema and reduced inflammatory cell influx by more than $90 \%$, and inhibitors of thiol proteinases also reduced emphysema and inflammatory cell infiltration. Neither class of proteinase inhibitor blocked IL-13-induced mucus metaplasia.

The full impact of Zheng's model is yet to be felt, but several points now seem clear. First, emphysema can be produced with minimal apparent involvement of neutrophils. Second, metalloproteinases, particularly MMP- 
9 and MMP-12, as well as thiol proteinases, may be responsible for alveolar septal destruction. Third, both resident lung parenchymal cells and inflammatory cells may contribute to lung injury during inflammation. Fourth, mucus metaplasia, a feature of chronic bronchitis, may be induced by inflammation in vivo, suggesting that the gap between asthma and COPD is not as wide as many have thought. In fact, the work of Zheng et al. (2) might suggest that the consequences of lung inflammation depend largely upon the site of the injury: if proximal airways are primarily affected, asthma could be the clinical outcome, whereas if the lung parenchyma is primarily affected, emphysema might predominate.

\section{Third-generation animal models: humanized mice}

Technology now allows targeted deletion of murine genes, followed by inserting (or "knocking in") human genes under control of the murine promoters. Now under development is a third generation of emphysema-prone mice in which the murine genes for $\alpha_{1}$ antitrypsin have been deleted and replaced with genes for either normal (Pi M) or deficient (Pi Z) human $\alpha_{1}$ antitrypsin. When successfully developed, these mice will allow direct testing of the possibility that deficiency of $\alpha_{1}$-antitrypsin results in inappropriate pulmonary inflammatory responses $(10,11)$, and they will also allow testing of new interventions, including hepatic gene repair. Such mice could be bred into a background deficient for leukocyte elastase, the cognate enzyme for $\alpha_{1-}$ antitrypsin, to test the prediction that doubly mutant mice will not differ from wild-type mice in their susceptibility to emphysema. Future mice could recapitulate other human effector systems of interest.

\section{Taking new findings to the clinic}

Regardless of the current and future successes of animal models of emphysema, new hypotheses and preventive therapies must eventually be tested in humans who have or are at risk for the disease. These are exciting times in which we are beginning to develop real insights into the complexities of the pathogenesis of emphysema, and in which real prospects for intervention loom. While high-resolution computerized tomography scanning offers the hope of an improved end point for following the course of the disease (12), intervention trials will necessarily be prolonged and expensive because of the slow progression of emphysema in humans. COPD, unfortunately, receives the least public research funding of any disease of adulthood, when adjusted for disease burden (13). Potential therapies that show promise in animal models may languish untested without infusion of more public funds.

\section{Acknowledgments}

This work was supported by Public Health Service Grant HL 46440.
1. Niewoehner, D.E. 1988. Cigarette smoking, lung inflammation and the development of emphysema. J. Lab. Clin. Med. 111:15-27.

2. Zheng, T., et al. 2000. Inducible targeting of IL-13 to the adult lung causes matrix metalloproteinase- and cathepsin-dependent emphysema. J. Clin. Invest. 106:1081-1093.

3. Campbell, E.J. 1986. Preventive therapy of emphysema: lessons from the elastase model. Am. Rev. Respir. Dis. 134:435-437.

4. Gross, P., Pfitzer, E.A., Tolker, E., Babyak, M.A., and Kaschak, M. 1965. Experimental emphysema: its production with papain in normal and silicotic rats. Arch. Environ. Health. 11:50-58.

5. Eriksson, S. 1964. Pulmonary emphysema and alpha $_{1}$-antitrypsin deficiency. Acta Med. Scand. 175:197-205.

6. Liou, T.G., and Campbell, E.J. 1996. Quantum proteolysis resulting from release of single granules by neutrophils: a novel, non-oxidative mechanism of extracellular proteolytic activity. $J$. Immunol. 157:2624-2631.

7. Keil, M., Lungarella, G., Cavarra, E., van Even, P., and Martorana, P.A. 1996. A scanning electron microscopic investigation of genetic emphysema in tight-skin, pallid, and beige mice, three different C57 BL/6J mutants. Lab. Invest. 74:353-362.

8. D’Armiento, J., Dalal, S.S., Okada, Y., Berg, R.A., and Chada, K. 1992. Collagenase expression in the lungs of transgenic mice causes pulmonary emphysema. Cell. 71:955-961.

9. Hautamaki, R.D., Kobayashi, D.K., Senior, R.M. and Shapiro, S.D. 1997. Requirement for macrophage elastase for cigarette smoke-induced emphysema in mice. Science. 277:2002-2004.

10. Hill, A.T., Campbell, E.J., Bayley, D.L., Hill, S.L., and Stockley, R.A. 1999. Evidence for excessive bronchial inflammation during an acute exacerbation of chronic obstructive pulmonary disease in patients with alpha(1)-antitrypsin deficiency (Pi Z). Am. J. Respir. Crit. Care Med. 160:1968-1975.

11. Hill, A.T., Bayley, D.L., Campbell, E.J., Hill, S.L., and Stockley, R.A. 2000. Airways inflammation in chronic bronchitis: the effects of smoking and $\alpha_{1}$ antitrypsin deficiency. Eur. Respir. J. 15:886-890.

12. Dirksen, A., et al. 1999. A randomized clinical trial of alpha(1)-antitrypsin augmentation therapy. Am. J. Respir. Crit. Care Med. 160:1468-1472.

13. Gross, C.P., Anderson, G.F., and Powe, N.R. 1999. The relation between funding by the National Institutes of Health and the burden of disease. $N$. Engl. J. Med. 340:1881-1887. 